6. Liu B, Havers FP, Zhou L, Zhong H, Wang X, Mao S, et al. Clusters of human infections with avian influenza A(H7N9) virus in China, March 2013 to June 2015. J Infect Dis. 2017;216 (Suppl_4):S548-S554. http://dx.doi.org/10.1093/infdis/jix098

7. Fang CF, Ma MJ, Zhan BD, Lai SM, Hu Y, Yang XX, et al. Nosocomial transmission of avian influenza A (H7N9) virus in China: epidemiological investigation. BMJ. 2015;351:h5765. http://dx.doi.org/10.1136/bmj.h5765

8. Chen H, Liu S, Liu J, Chai C, Mao H, Yu Z, et al. Nosocomial co-transmission of avian influenza A(H7N9) and A(H1N1)pdm09 viruses between 2 patients with hematologic disorders. Emerg Infect Dis. 2016;22:598-607. http://dx.doi.org/10.3201/eid2204.151561

9. Uyeki TM, Katz JM, Jernigan DB. Novel influenza A viruses and pandemic threats. Lancet. 2017;389:2172-4. http://dx.doi.org/10.1016/S0140-6736(17)31274-6

Address for correspondence: Qun Li, Public Health Emergency Center, Chinese Center for Disease Control and Prevention, Beijing, 102206, China; email: liqun@chinacdc.cn

\section{Human African \\ Trypanosomiasis in Emigrant Returning to China from Gabon, 2017}

\author{
Xinyu Wang, ${ }^{1}$ Qiaoling Ruan, ${ }^{1}$ Bin $X u,{ }^{1}$ \\ Jianfei Gu, Yiyi Qian, Muxin Chen, Qin Liu, \\ Qing Lu, Wenhong Zhang
}

Author affiliations: Fudan University, Shanghai, China (X. Wang, Q. Ruan, B. Xu, J. Gu, Y. Qian, Q. Lu, W. Zhang); Chinese Center for Disease Control and Prevention, WHO Collaborating Center for Tropical Diseases, Shanghai (M. Chen, Q. Liu)

DOI: https://doi.org/10.3201/eid2402.171583

Human African trypanosomiasis is endemic to parts of subSaharan Africa and should be considered in the differential diagnosis of patients who have visited or lived in Africa. We report a 2017 case of stage 2 Trypanosoma brucei gambiense disease in an emigrant who returned to China from Gabon.

$\mathrm{H}$ uman African trypanosomiasis (HAT), or sleeping sickness, is a tsetse fly-borne parasitic disease that is endemic to parts of sub-Saharan Africa. In central and west Africa, Trypanosoma brucei gambiense causes the slow-progressing form of the disease, and T. brucei rhodesiense causes the fast-

${ }^{1}$ These authors contributed equally to this article. progressing form in east and southern Africa (1). We report a confirmed case of HAT, after a probable tsetse fly bite, in a man who returned to China from Gabon in central Africa.

A previously healthy 60 -year-old man from China lived in Gabon for 8 years. He served as a seaman and traveled between Libreville and Kango to transport river sand. In July 2016, when he was working on a rural farm in Libreville, he had a painful, unidentified insect bite on his right lower limb. The bite wound developed into an indurated, erythematous, and painful skin lesion. He received antiviral and antityphoid therapy in Gabon. Although the skin lesion healed, he had intermittent fever (up to $40^{\circ} \mathrm{C}$ ), headache, and fatigue.

He returned to Jiangshu, China, for further treatment in June 2017. Magnetic resonance imaging (MRI) of the brain revealed temporal foci suggestive of white matter demyelination. Brain magnetic resonance angiography and electroencephalography revealed normal findings. He had daytime somnolence 2 weeks before admission to Huashan Hospital, associated with Fudan University in Shanghai, on August 30. The patient was lethargic during admission and had a temperature of $38.5^{\circ} \mathrm{C}$ and palpable cervical and inguinal lymph nodes. Hyperpigmentation of the right lower limb was visible. Meningeal irritation and the Babinski sign were absent.

The preliminary diagnosis was suspected HAT. We performed bone marrow puncture, which revealed a few trypomastigotes (Trypanosoma spp.; Figure 1, panel A). We also found trypanosomes in a peripheral blood smear. We sent a serum sample to the Chinese Center for Disease Control and Prevention (China CDC), which showed a positive result for the T. brucei gambiense antibody test. A cerebrospinal fluid (CSF) sample revealed an open pressure of $15 \mathrm{~cm} \mathrm{H}_{2} \mathrm{O}$, a leukocyte count of 9 cells $/ \mu \mathrm{L}$, a protein level of $1,412 \mathrm{mg} /$ $\mathrm{dL}$, and a glucose level of $1.6 \mathrm{mmol} / \mathrm{L}$. Direct examination revealed no trypanosomes in the CSF, although next-generation sequencing identified T. brucei gambiense in the CSF and bone marrow (stage 2 disease). Brain MRI revealed hyperintense signal changes in the left basal ganglia, and positron emission tomography-computed tomography suggested reduced glucose metabolism (Figure 1, panels B, C). The World Health Organization (WHO) and China CDC helped obtain nifurtimox and eflornithine, which we administered to the patient within $48 \mathrm{~h}$ after the diagnosis. The patient was discharged after 10 days of treatment.

Several HAT cases had previously been imported into China. One case-patient was a 45-year-old man who worked in forests and river valleys in Gabon and was diagnosed with T. brucei gambiense disease by blood smear in 2014, two months after returning to China (2). The second case involved a woman, 41 years of age, who traveled to Tanzania and Kenya, and was diagnosed with T. brucei rhodesiense by blood smear in 2017, one week after returning to China. Both cases were confirmed by molecular 


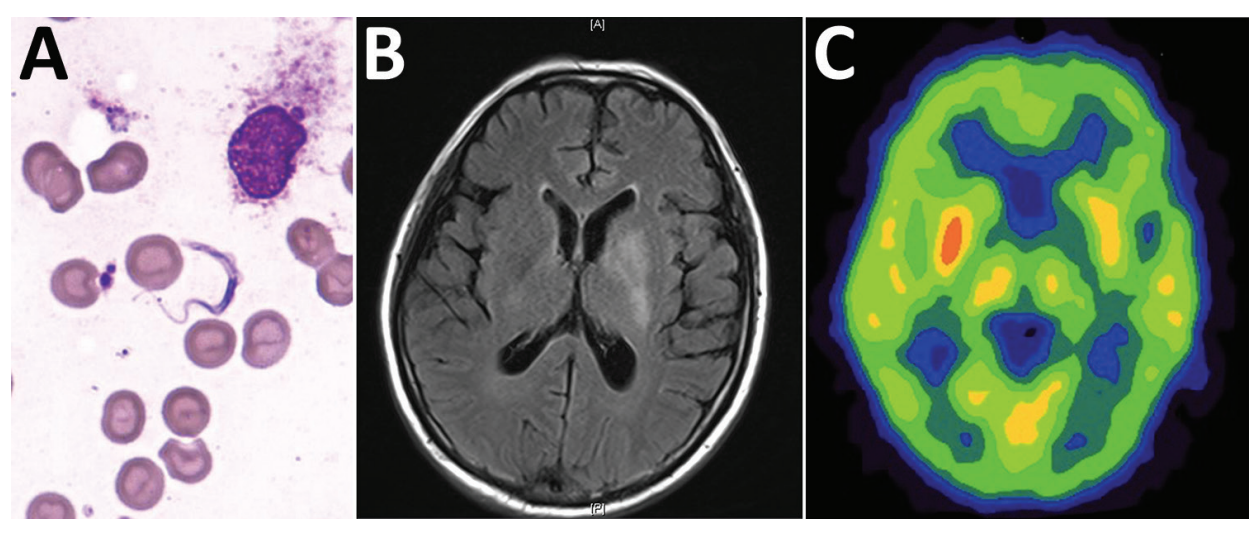

Figure. Bone marrow test results and brain imaging of a 60 -yearold man who returned to China from Gabon with suspected human African trypanosomiasis. A) Trypanosoma spp. (later determined to be $T$. brucei gambiense) in a Giemsa-stained thin bone marrow film. Original magnification $\times 1,000$. B) $A$ T2-weighted fluid-attenuated inversion recovery image with hyperintense signal changes in the left basal ganglia. C) Brain positron emission tomographycomputed tomography suggested reduced glucose metabolism in the left basal ganglia.

diagnosis. Additional cases may have been imported to China, but we could not find any reports after a review of literature and available national records.

The latest data from WHO indicate that new HAT cases have declined during the past 15 years, reaching a low of 2,184 cases in 2016 (3). Nevertheless, HAT cases exported from Africa have been reported in all continents. In non-HAT-endemic areas, 94 cases of HAT were reported during 2000-2010, including 26 cases of T. brucei gambiense disease (4). T. brucei rhodesiense disease typically involves tourists who have visited national parks and game reserves in eastern and southern Africa, whereas T. brucei gambiense disease is rarer and typically involves migrants and long-term emigrants ( 1 ). Persons in Gabon are at risk for T. brucei gambiense infection; 403 cases were reported during 2010-2014 (4). Nine exported HAT cases from Gabon have been reported, including 5 from Libreville; all these cases were T. brucei gambiense disease, and 6 were in immigrants from various countries $(2,5-7)$.

Although HAT is uncommon in nonendemic settings, it should be included in the differential diagnosis of travelers who return from sub-Saharan Africa with fever. T. brucei rhodesiense disease causes acute illness and usually manifests as severe parasitemia; thus, the diagnosis is easier and quicker. In contrast, T. brucei gambiense disease usually has a chronic progressive course, with an estimated untreated infection duration of 2 years (8). Blood film review is essential for the early detection of HAT. Moreover, T. brucei gambiense infection can be reliably diagnosed by testing for specific antibodies, although this test is not available in most areas of China. As in this case, next-generation sequencing technologies are useful for diagnosing unknown tropical febrile illnesses (9). Because rapid diagnosis and treatment of HAT are essential, countries with large populations of travelers to HAT-endemic regions should maintain diagnostic tools and appropriate medication to facilitate rapid clinical management.

\section{Acknowledgments}

We thank Gerardo Priotto and Jose Ramon Franco for providing the nifurtimox/eflornithine medication and advice regarding the patient's management. We also express our thanks to Xiao-nong Zhou, Jia-xu Chen, and Xun-jia Chen.

\section{About the Authors}

Dr. Wang is an attending physician in the Department of Infectious Disease, Huashan Hospital, affiliated with Fudan University; primary research interest is travel medicine research. Dr. Ruan is a resident physician in the Department of Infectious Disease, Huashan Hospital, affiliated with Fudan University; primary research interests are infectious diseases, especially tuberculosis. Dr. Xu is an attending physician of Department of Infectious Disease, Huashan Hospital, affiliated with Fudan University; primary research interest is the management of severe infectious diseases.

\section{References}

1. Büscher P, Cecchi G, Jamonneau V, Priotto G. Human African trypanosomiasis. Lancet. 2017;11:2397-2409. http://dx.doi.org/10.1016/S0140-6736(17)31510-6

2. Hu X, Sun Y, Xia X, Xu L, Min B, Zhou D, et al. Photo quiz: A man with lymphadenopathy and lethargy. J Clin Microbiol. 2016;54:2631. http://dx.doi.org/10.1128/JCM.03285-14

3. World Health Organization. Eliminating sleeping sickness as a public health problem is on track [cited 2017 Sept 19] http://www.who.int/trypanosomiasis_african/news/ HAT_elimination_on_track/en/

4. Franco JR, Cecchi G, Priotto G, Paone M, Diarra A, Grout L, et al. Monitoring the elimination of human African trypanosomiasis: Update to 2014. PLoS Negl Trop Dis. 2017;11:e0005585. http://dx.doi.org/10.1371/journal.pntd.0005585

5. Simarro PP, Franco JR, Cecchi G, Paone M, Diarra A, Ruiz Postigo JA, et al. Human African trypanosomiasis in non-endemic countries (2000-2010). J Travel Med. 2012;19:44-53. http://dx.doi.org/10.1111/j.1708-8305.2011.00576.x 
6. Simon F, Mura M, Pagès F, Morand G, Truc P, Louis F, et al. Urban transmission of human African trypanosomiasis, Gabon. Emerg Infect Dis. 2012;18:165-7. http://dx.doi.org/10.3201/eid1801.111384

7. Migchelsen SJ, Büscher P, Hoepelman AI, Schallig HD, Adams ER Human African trypanosomiasis: a review of non-endemic cases in the past 20 years. Int J Infect Dis. 2011;15:e517-24. http://dx.doi.org/10.1016/j.ijid.2011.03.018

8. Checchi F, Funk S, Chandramohan D, Haydon DT, Chappuis F. Updated estimate of the duration of the meningo-encephalitic stage in Gambiense human African trypanosomiasis. BMC Res Notes. 2015;8:292. http://dx.doi.org/10.1186/s13104-015-1244-3

9. Lefterova MI, Suarez CJ, Banaei N, Pinsky BA. Next-generation sequencing for infectious disease diagnosis and management: a report of the Association for Molecular Pathology. J Mol Diagn. 2015;17:623-34. http://dx.doi.org/10.1016/j.jmoldx.2015.07.004

Address for correspondence: Wenhong Zhang, Huashan Hospital, Department of Infectious Diseases, Fudan University, 12 M. Wulumuqi Rd, Shanghai 200040, China; email: zhangwenhong@fudan.edu.cn

\section{Dengue-Associated Posterior Reversible Encephalopathy Syndrome, Vietnam}

\section{Nguyen Thi Hoang Mai, Nguyen Hoan Phu, Ho Dang Trung Nghia, Tran My Phuong, Du Trong Duc, Nguyen Van Vinh Chau, Bridget Wills, Choie Cheio Tchoyoson Lim, Guy Thwaites, Cameron Paul Simmons, Sophie Yacoub}

\begin{abstract}
Author affiliations: Hospital for Tropical Diseases, Ho Chi Minh City, Vietnam (N.T.H. Mai, N.H. Phu, H.D.T. Nghia, T.M. Phuong, D.T. Duc, N.V.V. Chau); Oxford University Clinical Research Unit, Wellcome Trust Major Overseas Programme, Ho Chi Minh City (N.T.H. Mai, N.V.V. Chau, B. Wills, G. Thwaites, C.P. Simmons, S. Yacoub); University of Oxford, Oxford, UK (B. Wills, G. Thwaites); National Neuroscience Institute, Singapore (C.C.T. Lim); University of Melbourne, Melbourne, Victoria, Australia (C.P. Simmons); Imperial College London, Hammersmith Campus, London, UK (S. Yacoub)
\end{abstract}

DOI: https://doi.org/10.3201/eid2402.171634

Dengue can cause neurologic complications in addition to the more common manifestations of plasma leakage and coagulopathy. Posterior reversible encephalopathy syndrome has rarely been described in dengue, although the pathophysiology of endothelial dysfunction likely underlies both. We describe a case of dengue-associated posterior reversible encephalopathy syndrome and discuss diagnosis and management.
$\mathrm{O}^{\prime \prime}$ December 20, 2015, a 55-year-old woman in Vietnam sought medical care at her local hospital near Ho Chi Minh City with 1 day of fever, muscle aches, and anorexia. She had no remarkable medical history; she did not take any medications and had not received any recent vaccinations. Initial hematology and biochemistry tests were normal, but a rapid test for dengue nonstructural protein 1 antigen was positive. She remained hemodynamically stable and did not experience any bleeding or have evidence of plasma leakage. On December 24, she had a generalized convulsion and was transferred to the intensive care unit at the Hospital for Tropical Diseases.

At admission, her Glasgow Coma Scale score was $10 / 15$ (eyes 3 , motor 4 , voice 3 ); she was confused, and speech was slurred. Her temperature was $37.5^{\circ} \mathrm{C}$, heart rate was 100 beats $/ \mathrm{min}$, blood pressure was $140 / 90$ $\mathrm{mmHg}$, and respiratory rate was 18 breaths $/ \mathrm{min}$. Cardiorespiratory and abdominal examinations were normal. Neurologic examination demonstrated increased tone in upper and lower limbs and bilateral upgoing plantar reflexes; assessment of power was difficult because of generalized rigidity. Examination of her eye movements demonstrated vertical gaze palsy, but other cranial nerves were intact.

Blood tests showed hemoglobin level of $14 \mathrm{~g} / \mathrm{dL}$, hematocrit level of $42.2 \%$, a white cell count of $11 \times 10^{9}$ cells $/ \mathrm{L}$, and a platelet count of $100 \times 10^{9} / \mathrm{L}$. A test for dengue nonstructural protein 1 antigen remained positive, but PCR was negative. Urea and electrolyte levels were normal, but liver transaminases showed elevated aspartate aminotransferase of $386 \mathrm{U} / \mathrm{L}$ and alanine aminotransferase of $242 \mathrm{U} / \mathrm{L}$. Chest radiograph was unremarkable, but a brain computed tomography scan demonstrated bilateral cerebral and cerebellar white matter hypodensities. Lumbar puncture demonstrated an opening pressure of 12 $\mathrm{cm} \mathrm{H}_{2} \mathrm{O}$, a cerebrospinal fluid (CSF) cell count of 7 cells/ $\mu \mathrm{L}$, a red cell count of 4 cells $/ \mu \mathrm{L}$, protein level of $4.4 \mathrm{~g} /$ $\mathrm{dL}$, glucose level of $4.76 \mathrm{mmol} / \mathrm{L}(10.4 \mathrm{mmol} / \mathrm{L}$ in blood), and lactate level of $3.25 \mathrm{mmol} / \mathrm{L}$. Dengue virus IgM was detected in CSF; Japanese encephalitis virus IgM was negative. CSF dengue and herpes simplex virus PCR were negative. Microbiologic cultures performed on blood and CSF were sterile.

Magnetic resonance imaging (MRI) of the brain, performed the following day, demonstrated bilateral symmetric high signal on T2-weighted and fluid attenuation inversion recovery images, involving periventricular and deep cerebral white matter (Figure, panel A). The differential diagnoses included encephalitis or acute demyelinating encephalomyelitis (ADEM), and a course of intravenous methylprednisolone was started $(1 \mathrm{~g} / \mathrm{d})$. After 5 days, this regimen was converted to oral prednisolone $(60 \mathrm{mg} / \mathrm{d})$, tapering over 5 days. Phenobarbital was administered for 1 